ACCEPTED MANUSCRIPT

\title{
Weak antilocalization effect in exfoliated black phosphorus revealed by temperature- and angle-dependent magnetoconductivity
}

To cite this article before publication: Zhipeng Hou et al $2018 \mathrm{~J}$. Phys.: Condens. Matter in press https://doi.org/10.1088/1361-648X/aaa68e

\section{Manuscript version: Accepted Manuscript}

Accepted Manuscript is "the version of the article accepted for publication including all changes made as a result of the peer review process, and which may also include the addition to the article by IOP Publishing of a header, an article ID, a cover sheet and/or an 'Accepted

Manuscript' watermark, but excluding any other editing, typesetting or other changes made by IOP Publishing and/or its licensors"

This Accepted Manuscript is @ 2018 IOP Publishing Ltd.

During the embargo period (the 12 month period from the publication of the Version of Record of this article), the Accepted Manuscript is fully protected by copyright and cannot be reused or reposted elsewhere.

As the Version of Record of this article is going to be / has been published on a subscription basis, this Accepted Manuscript is available for reuse under a CC BY-NC-ND 3.0 licence after the 12 month embargo period.

After the embargo period, everyone is permitted to use copy and redistribute this article for non-commercial purposes only, provided that they adhere to all the terms of the licence https://creativecommons.org/licences/by-nc-nd/3.0

Although reasonable endeavours have been taken to obtain all necessary permissions from third parties to include their copyrighted content within this article, their full citation and copyright line may not be present in this Accepted Manuscript version. Before using any content from this article, please refer to the Version of Record on IOPscience once published for full citation and copyright details, as permissions will likely be required. All third party content is fully copyright protected, unless specifically stated otherwise in the figure caption in the Version of Record.

View the article online for updates and enhancements. 
Weak antilocalization effect in exfoliated black phosphorus revealed by temperatureand angle-dependent magnetoconductivity

Zhipeng $\mathrm{Hou}^{1+}$, Chen Gong ${ }^{2+}$, Yue Wang ${ }^{1}$, Qiang Zhang ${ }^{2}$, Bingchao Yang ${ }^{3}$, Hongwei Zhang ${ }^{1}$, EnKe Liu ${ }^{1}$, Zhongyuan $\mathrm{Liu}^{2}$, Zhongming Zeng ${ }^{4}$, Guangheng $\mathrm{Wu}^{1}$, Wenhong Wang1,*, and Xi-xiang Zhang ${ }^{2 \dagger}$

${ }^{1}$ Institute of Physics, State Key Laboratory for Magnetism, Beijing National Laboratory for Condensed Matter Physics, Chinese Academy of Sciences, Beijing 100190, China

${ }^{2}$ King Abdullah University of Science and Technology (KAUST), Physical Science and Engineering, Thuwal 23955-6900, Saudi Arabia.

${ }^{3}$ State Key Laboratory of Metastable Materials Science and Technology, Yanshan University, Qinhuangdao 066004, China

${ }^{4}$ Key Laboratory of Nanodevices and Applications,Suzhou Institute of Nano-tech and Nano-bionics, Chinese Academy of Sciences, Ruoshui Road 398, Suzhou 215123, China

Correspondence should be addressed to W.H.W and X. X. Zhang (email. wenhong.wang@iphy. ac. cn; xixiang.zhang@kaust. edu. sa)

${ }^{+}$These authors contributed equally to this work

\section{Abstract}

Recently, there have been increasingly heated debates on whether there exists a surface resonance state (SRS) in black phosphorus (BP), as suggested by recent angle-resolved photoemission spectroscopy (ARPES) results. To unambiguously resolve this issue, we have performed temperature- and angle-dependent magnetoconductivity measurements on exfoliated, high-quality BP single crystals. A pronounced weak-antilocalization (WAL) effect was observed within a narrow temperature range of $8-16 \mathrm{~K}$, with the electrical current flowing parallel to the cleaved $a c$-plane (along the $a$ - or $c$-axis) and the magnetic field along the $b$-axis. The angle-dependent magnetoconductivity and the Hikami-Larkin-Nagaoka (HLN) model-fitted results have revealed that the observed WAL effect shows obvious surface-bulk coherent features, which. This strongly supports the existence of SRS in black phosphorus. 


\section{Introduction}

Phosphorus is a light, pentavalent element which exists in a number of allotropic forms. As the most stable form of phosphorus under normal conditions, orthorhombic black phosphorus (BP) has a layered structure, much like graphene and transition metal dichalcogenides (TMDs), though its atoms are puckered (see Fig.1a). Extensive theoretical and experimental investigations have revealed that numerous intriguing physics can be realized in this material, such as large linear magnetoresistance (MR) [1], high in-plane anisotropy [2-4], high carrier mobility [5,6], strain-induced gap modifications [7,8], semiconductor-metal transition [9,10], and superconductivity under high pressure [11]. In addition, its unique band structure and transport properties have driven a flurry of exciting researches trying to probe the promising applications in further nanoelectronics and nanophotonics [5,6,12-14]. For example, BP has a tunable, thickness-dependent direct band gap, varying from $\sim 0.3 \mathrm{eV}$ (bulk) to $>1.4 \mathrm{eV}$ (monolayer), which is valuable for developing novel optical and other devices with functionalities based on heterostructures with two-dimensional (2D) materials [13, 14]. The field-effect transistor (FET) based on BP has been reported to exhibit superior semiconductor device performance [6,15-18]; our recent results show that $\mathrm{BP}$ can serve as the electrode material for all-solid-state supercapacitors [19].

Despite the numerous intriguing properties presented by BP, its electronic structures are not yet completely understood. Previous numerical work on bulk BP has shown that it has a usual narrow-gapped semiconductor with a $0.3 \mathrm{eV}$ band gap at the $Z$ point $[13,14]$. However, recent angle-resolved photoemission spectroscopy (ARPES) results have demonstrated that there exist surface resonance states (SRS) near the top of the valence band along the [010] direction, indicating that the current theoretical methodologies do not fully describe the important electronic properties of BP $[20,21]$. In the field of nanoelectronics, surface effects like SRS and surface states (SS) have a critical role in the performance of electrical and optical devices, such as the prevention of mobility degradation and the Fermi-level pinning effect [22]. Thus, the study of SRS in relation to transport properties is not only of vital importance for understanding the relative contributions from bulk states and SRS near the Fermi level, but also crucial for the development of new electronic devices based on BP.

Similar to true surface states, surface resonance states are also localized at the surface of a 
material and have no $k_{\mathrm{z}}$ dispersion. The main difference between surface states and surface resonance states is that the surface state (SS) exists only in the projected bulk band gap, whereas the surface resonance state (SRS) is a hybrid between the 2D surface state and the three-dimensional (3D) bulk state (there is a substantial spatial overlap between their wave functions), and the SRS weakly embeds in the bulk states [23, 24]. From the electronic transport point of view, SRSs allow a charge to be transferred dynamically between the surface and the bulk. Therefore, the bulk and surface transport channels can coherently couple with each other, which makes the transport exhibit both bulk and surface characteristics, as is the case in Bi thin film [25]. To observe the SRS experimentally, we must reduce the interference of the bulk states to pick out the contributions from the SS in transport, which is a similar to the method used for the observation of pure surface states. For Bi, due to the semimetallic band structure in the bulk, its surface effects (SS and SRS) are usually observed in the thin film form, where the bulk-to-surface ratio has been reduced progressively [25-27]. However, as a semiconductor, bulk BP has a direct band gap of $0.2 \mathrm{eV}$, and its corresponding bulk conductivity decreases drastically at low temperatures (below $50 \mathrm{~K}$ ) $[10,28]$, which allow us to observe the coherent characteristics directly on the bulk BP at low temperatures. In addition, high-resolution synchrotron-based photo-electron diffraction (HRSPD) investigations suggest that the surface of a single crystalline bulk BP can be a good approximation of an isolated single layer [29]. Following the above lines of reasoning, we have performed magnetotransport measurements on exfoliated BP single crystals, over a temperature range of $2-20 \mathrm{~K}$ in a maximum external magnetic field of 7 tesla (T), to search for the evidence of possible surface-bulk coherent transport properties. A pronounced weak-antilocalization (WAL) effect was observed in the high-quality samples within a narrow temperature range of $8-16 \mathrm{~K}$, with the electrical current flowing parallel to the cleaved $a c$-plane (along the $a$ - or $c$-axis) and a magnetic field along the $b$-axis (The notations $a, b, c$ are connected with the crystallographic axis of the BP, as is shown in Fig. 1a. The $a$ - and $c$-axis is along the armchair and zigzag direction, respectively. The direction $b$-axis is normal to the cleaved ac-plane). More intriguingly, both the angle-dependent magnetoconductivity and Hikami-Larkin-Nagaoka (HLN) fitting results show obvious surface-bulk coherent features, which is at solid evidence for the existence of surface resonance states [25]. 
The BP crystals were synthesized from red phosphorous, as described in our previous work $[1,19]$. The as-prepared crystals were then cleaved at room temperature in an argon-filled glove box, in which the oxygen and humidity content were less than $0.1 \mathrm{ppm}$, resulting in a well-developed and shining (0l0) surface. To perform the transport measurements, a conventional Hall-bar was fabricated on the cleaved samples with a typical size of $0.6 \times 0.6 \times 0.08 \mathrm{~mm}^{3}$ (see Figure $1 \mathrm{~b}$ and $\mathrm{c}$ ). To protect the samples from oxidation during transfer and transport measurement, we covered the surface of the samples with varnish film. The magnetotransport measurements were performed on a Quantum Design PPMS-9 with a maximum magnetic field of $7 \mathrm{~T}$.

\section{Results and discussions}

Figure 1d shows the temperature-dependent longitudinal resistivity $\rho_{\mathrm{xx}}$ obtained on two representative, freshly cleaved samples (Sample A and B), from $2 \mathrm{~K}$ to $300 \mathrm{~K}$ under zero magnetic field. For both samples, $\rho_{\mathrm{xx}}$ increased exponentially as the temperature $T$ decreased below $50 \mathrm{~K}$, due to the freezing of impurity ionization, and did not show a trend of saturation, even at a low temperature of $2 \mathrm{~K}$. For example, the more resistive Sample A exhibits an extremely large value of $952 \Omega \mathrm{cm}$ at $2 \mathrm{~K}$, which is nearly 1,750 times larger than the resistivity at $50 \mathrm{~K}$. The greatly suppression of bulk conductivity in BP provides us an opportunity to observe the SRS by using the transport measurements at low temperature.

To search for the signs of SRS, we performed magnetotransport measurements in a magnetic field up to $7 \mathrm{~T}$ over a temperature range of $2 \mathrm{~K}$ to $20 \mathrm{~K}$ for both the two samples. Figure $2 \mathrm{a}$ shows the field-dependent magnetoresistance (MR) curves (MR is defined as $[\rho(\mathrm{H})-\rho(0)] / \rho(0)] \times 100 \%$, where $\rho(H)$ and $\rho(0)$ are the resistivity at field $H$ and zero, respectively) at different temperatures for Sample A, with the current flowing along the $c$-axis in the $a c$-plane and the magnetic field along the $b$-axis. Notably, the in-plane crystallographic orientations were determined by Electron back-scattered diffraction (EBSD) pattern, as shown in our previous reports [30]. The behavior of MR obtained with the current along the $a$-axis in the $a c$-plane is similar to the MR behavior with the current flowing along the $c$-axis, except for the value of MR [1]. It is interesting to note that the behavior of MR in Figure $2 \mathrm{a}$ can be divided into two regions, i.e., high-field $(> \pm 4 \mathrm{~T})$ and low-field $(< \pm 4 \mathrm{~T})$ regions. In the high-field region, MR increases linearly or quadratically with the increase of external magnetic field, owing to the mobility fluctuation, or Lorentz effect, as reported in our previous work [1]. In the low-field region, the MR curves display negative cusps in 
the temperature range 2-8_K, which is reminiscent of the weak localization (WL) effect that results from the constructive interference of two time-reversal electron trajectories [31-35]. Usually, a WL effect occurs at low temperatures when thermal disturbance is significantly suppressed and electrons are scattered elastically with preserved phase coherence [31-35]. In the case of BP, the appearance of the WL effect can be attributed to the dominance of elastic scattering caused by impurities [36,37]. With increasing temperature, the WL effect gradually disappears; instead,/a sharp positive cusp was observed in the low-field region over the temperature range $8 \mathrm{~K}$ to $16 \mathrm{~K}$ (see Fig. S1). This feature is regarded as an obvious signature of the weak-antilocalization (WAL) effect [38-41]. It should be noted here that WAL was not unique to this particular Sample A but was also observed in Sample B (as shown in Fig. S2). In contrast to WL, WAL arises in a phase-coherent conductor when destructive interference is formed between two time-reversal electron paths. It is usually observed in materials with strong spin-orbital coupling (SOC), such as Bi [25], InAs [42], and topological insulators [31, 35, 38-41]. Since phosphorus is a light element, the SOC should not be strong enough to result in WAL $[8,12]$. Therefore, WAL cannot be explained by the effect of SOC in BP. As is known to us, MR in the nonmagnetic materials is dominated by the orbital effects originating from the cyclotron motion of electrons due to the Lorentz force. According to the two-band model, MR can be scaled with $\mathrm{MR} \sim\left(w_{\mathrm{c}} \tau\right)^{2} \sim(\mathrm{e} \tau /$ $\left.m^{*}\right) B^{2} \sim \mu^{2} B^{2} \quad\left(\tau\right.$ is the scattering time, $m^{*}$ is the effective mass, and $B$ is the magnetic field; $w_{\mathrm{c}}\left(=\mathrm{eB} / m^{*}\right)$ is the cyclotron's frequency). Thus, the behavior of MR is closely related to the band structure along the direction of magnetic field. Based on the results above, it is preliminarily supposed that the observed WAL effect is related to the special band structure along the $b$-axis.

To investigate the effect of band structure along $c$-axis on the MR, we measured the field dependence of MR at different temperatures for Sample A (see Fig. 2b), with the current flowing along the $b$-axis and the magnetic field applied parallel to the ac-plane (the corresponding curves are shown in Fig. S3). Since the sample along the $b$-axis is quite thin, we cannot use the standard 4-point configuration but the method described in the reference 42 (the electric configuration is shown in the inset of Fig. 2b). Surprisingly, no WAL was observed in this configuration; the WL effect was clearly observed, which strongly indicates that in BP, WAL is anisotropic (plane-dependent), but WL is isotropic (plane-independent). Since WL results from the elastic 
scatterings of electrons at defects or imperfections in a material, it is easy to understand its isotropic feature (plane-independence). However, the WAL effect was observed only when the current was flowing along the $a c$-plane (either along $a$-axis or $c$-axis) with the magnetic field applied along the $b$-axis. This unique characteristic of WAL strongly suggests that the WAL in BP does not stem from the SOC interaction, but rather may be closely related to the special electrical band structure along the [010] direction, e.g. a surface resonance state, SRS.

As mentioned above, when SRS exists in a material, the coherent coupling between the surface and bulk conduction channels leads to a transport that shows both surface (2D) transport and bulk (3D) transport characteristics [25]. Now, let us examine first whether the observed WAL effect has the 2D characteristics. We explore how the field-dependent MR varies with the tilt angle $\theta$ between $H$ and the $b$-axis in magnetic fields up to $7 \mathrm{~T}$ for Sample A (the sketch is shown in Figure 3a). In a 2D transport system, the amplitude of MR depends only on the perpendicular component of the applied magnetic field $H \cos \theta$ to the $2 \mathrm{D}$ plane. The curves shown in Figure $3 \mathrm{a}$ represent the MR data measured at $10 \mathrm{~K}$ with different angles. It is evident that the value of MR decreases significantly with increasing $\theta$ over the whole magnetic field range, but particularly in the low-field region. For example, the MR obtained in $1 \mathrm{~T}$ at $0^{\circ}$ is nearly 6 times larger than that at $90^{\circ}$ (here MR is maximized at $\theta=0^{\circ}$ ). To show the relationship between MR and the perpendicular component of a magnetic field more clearly, we plotted the MR curves as a function of $H \cos \theta$ in Figure 3b. Very interestingly, all the curves obtained at different angles merge into a single curve when the perpendicular magnetic field $H \cos \theta$ is smaller than $1 \mathrm{~T}$, below which the WAL effect dominates the electrical transport. This strongly suggests that the WAL in BP is $2 \mathrm{D}$ in nature. Moreover, as shown in Figure 3c, when the external magnetic field rotates to the angle $\theta=90^{\circ}(H$ is parallel to the $a c$-plane and perpendicular to the current), the WAL effect disappears as surface channels are nolonger present to contribute to the MR effect, which is also consistent with a $2 \mathrm{D}$ nature. Further, we have performed the angle-dependent transport measurements at $11 \mathrm{~K}$ and $2 \mathrm{~K}$ (see Fig. S4). It is found that the WAL observed at $11 \mathrm{~K}$ is also 2D in nature, whereas the WL at 2 $\mathrm{K}$ shows a 3D feature. Based on the results, we can atready-perceive that the WAL effect in bulk BP is dominated by the surface transport.

To gain a deeper understanding of the WAL effect in BP, we analyze the data more quantitatively by fitting the data to the Hikami-Larkin-Nagaoka (HLN) model [43]: 


$$
\Delta G(H)=\frac{\alpha_{0} e^{2}}{2 \pi^{2} \hbar}\left[\psi\left(\frac{1}{2}+\frac{\hbar}{4 e H L_{\varphi}}\right)-\ln \left(\frac{\hbar}{4 e H L_{\psi}}\right)\right],
$$

where $\Delta G=G(\mathrm{H})-G(0), G(\mathrm{H})$ and $G(0)$ are the respective conductivity at magnetic field $H$ and zero, $\psi$ is the digamma function, $L_{\varphi}$ is the phase coherence length, and the coefficient $\alpha_{0}$ is the estimated number of independent channels contributing to the interference: $\alpha_{0}=-0.5$ for a single channel. In the Equation 1, we assume one dephasing rate to fit the WAL effect. However, at $8 \mathrm{~K}$, the field-dependence MR curve shows a coexistence of WAL (in the low field region) and WL (in the high field region). To fit the magnetoconductance which exhibits both WAL and WL at $8 \mathrm{~K}$, we assume one dephasing rate for WAL and another one for WL. Therefore, the HLN model can be modified as follow [35]:

$$
\Delta G(H)=\sum_{i=0,1} \frac{\alpha_{i} e^{2}}{\pi \mathrm{h}}\left[\psi\left(\frac{1}{2}+\frac{\hbar}{4 e H L_{\varphi \mathrm{i}}}\right)-\ln \left(\frac{\hbar}{4 e H L_{\varphi \mathrm{i}}}\right)\right]
$$

where the coefficients $\alpha_{0}$ and $\alpha_{1}$ stand for the weights of WAL and WL contributions, respectively, and $L_{\varphi i}$ is the corresponding phase coherence length. In Figure 4a, we present the low-field $\Delta G$ at temperatures ranging from $8 \mathrm{~K}$ to $12 \mathrm{~K}$. It is evident that the data of $\Delta G$ can be well described by the HLN model. The corresponding values of $\alpha_{0}$ and $L_{\varphi}$ for WAL were then obtained from the fitting as shown in Figure $4 b$ and Figure 4c, respectively. As shown in Figure 4b, the value of $L_{\varphi}$ decreases continuously with increasing temperature, indicating that WAL weakens as temperature increases. The value of $L_{\varphi}$ for WAL can be expressed as: $L_{\varphi}=\sqrt{D \tau_{\varphi}}$, where $D$ is the diffusion constant and $\tau_{\varphi}$ is the dephasing time. As shown in Figure $4 \mathrm{~b}$, the temperature-dependent $L_{\varphi}$ is fitted well with a power law of $L_{\varphi} \propto T^{-0.1}$. This power law leads to an effective exponent of temperature $p=0.2$ for the dephasing time, $\tau_{\varphi} \propto T^{-\mathrm{p}}$; this is considerably lower than the expected value of 1 for the quasielastic Nyquist electron-electron scattering process in $2 \mathrm{D}$, and indicates the existence of a weakly temperature-dependent carrier dephasing process. On the other hand, as shown in Figure 4c, a negative $\alpha_{0}$ as large as -16.7 was obtained at $16 \mathrm{~K}$, which greatly deviates from the theoretical values of -0.5 or -1 . This could be attributed to the dominant contribution of the bulk at high temperatures, as observed in half-Heusler topological insulators such as ScPtBi or LuPdBi $[39,40]$. As the temperature decreases, the bulk conductivity decreases dramatically, whereas the surface conducting channels play a more important role in the total conductivity leading to gradual reduction in the absolute value of $\alpha_{0}$. At $9 \mathrm{~K}$, we can obtain an absolute value of $\alpha_{0}$ as large as 0.58 , which agrees with the theoretical value of $\alpha_{0}$ and confirms 
the 2D-dominated WAL effect.

Actually, the total measured conductance of the sample should combine both the surface and bulk contributions. As shown in Figure 3a, the non-zero MR at $\theta=90^{\circ}$ strongly indicates the existence of bulk channels. Therefore, we must ask why the low-field MR curves at different angles still overlap and why the absolute value of $\alpha_{0}$ is still close to 0.5 . This question can be answered by considering the bulk-surface coherent transport, which makes the bulk and surface channels coherently couple with each other and form a single transport channel [25]. This model can also explain the deviation of the MR curve from the $H \cos \theta$ curve in the high-field regime. As shown in Figure 3b, when the magnetic field increases above 2 T, the external magnetic field breaks the time-reversal symmetry, leading to the strong suppression of the 2D-dominated WAL effect. In this scenario, due to the weakening of 2D channels, the 3D bulk channels decouple from the surface-bulk coupling. Therefore, we obtain a 3D-dominated MR in the high-field region and MR and $H \cos \theta$ curves which deviate from each other. Based on the results above, we found that both the angle-dependent magnetoconductivity and the HLN fitting results show obvious surface-bulk coherent features. This is strongly regarded as an powerfulevidence for the existence of SRS in BP.

Another issue to be addressed is the extremely small absolute value of $\alpha_{0}\left(\alpha_{0}=0.05\right)$ obtained at $8 \mathrm{~K}$. This is closely related to competition between the WAL and WL effects. Since WL gives a positive coefficient $\alpha_{1}$ and the WAL effect was weakened significantly by the WL effect, the deduced value of $\alpha_{0}$ was significantly reduced [30,34]. The crossover between the WAL and WL effects has also been observed in another 2D material, graphene, in which the transition occurs because elastic scattering weakens the chiral nature of Dirac fermions [31-33]. In this work, the WAL effect was not detected in all of the samples, though they were cleaved from the same crystal (see Fig. S5). We further found that the WAL effect in BP can only be observed in relatively high-quality samples that have fewer impurities. The WAL effect is strongly suppressed as the sample quality declines (see the Discussion Section in the Supplementary Materials). It is supposed that the origin of the suppression of WAL in BP and graphene is similar, though they possess completely different band structure. As is known to us, graphene is a semimetal and exhibits a metallic temperature dependence of the resistivity. Its band structure is completely different from that of the semiconductor BP. However, no matter grapheme or BP, at the low 
temperature, the impurity scattering plays an important role in their transport of electrons. In graphene, if the density of impurities reaches a certain values, the impurities scattering can make the electron obtain $2 \pi$ phase, which breaks the $\pi$ Berry phase of the band structure and makes quantum interference gradually change from destructive (WAL) to constructive (WL). In the case of BP, at the low temperatures, the impurity ionization dominates the transport of electrons and the obvious three-dimensional WL effect is observed below $8 \mathrm{~K}$. In the temperature range of 9-16 $\mathrm{K}$, the SRS dominated the transport behaviors of electrons and the possible $\pi$ phase results in a WAL effect. With the decrease of temperature, the role of impurities becomes more and more important, which makes the phase of electron gradually transform from $\pi$ to $2 \pi$ and therefore a crossover from WAL to WL. On the other hand, in the temperature range of 9-16 K, only WAL effect is observed and we fit the field-dependent MR curves assuming one phase coherence relaxation time. In the case of $8 \mathrm{~K}$, the MR curve shows a coexistence of WAL and WL and we fit it by assuming two relaxation times: one is for WL and the other one is for WAL. By using the equation 2, we can divide the relaxation time of WAL (in the low-field region) from that of WL (in the relatively high field region). Actually, we just assume only one relaxation time for WAL effect and therefore it is similar to that in graphene. Based on the results above, we supposed that the suppression of WAL in BP is elosely-related to elastic scattering (impurities, dislocations, or defects scattering) in BP, which may break the possible $\pi$ Berry phase or produce an effective random magnetic field that destroys the interference, similar to the case in graphene [34].

\section{Conclusions}

In summary, we have observed surface-bulk coherent features by performing angle-dependent magnetotransport measurements on exfoliated in a perpendicular to the $(0 l 0)$ surface within a narrow temperature range of $8-16 \mathrm{~K}$, where the bulk conductivity decreases drastically. This result presents a possible strong evidence for the existence of SRS. The key to observing this feature in bulk BP is using relatively high quality samples of BP. The surface transport can be strongly suppressed by scattering caused by impurities (elastic scattering) which results in bulk transport. Our results suggest that the SRS near the Fermi Level could play a key role in electronic transport when the bulk conductivity is greatly suppressed, and therefore could pave the way for developing further BP-based nanoelectronic devices. 


\section{Acknowledgments}

This work was supported by National Natural Science Foundation of China, NSFC (Grant Nos. 11474343 and 11574374), King Abdullah University of Science and Technology (KAUST) Office of Sponsored Research (OSR) under Award No: CRF-2015-2549-CRG4, China Postdoctoral Science Foundation NO. Y6BK011M51, and Strategic Priority Research Program B of the Chinese Academy of Sciences under the grant No. XDB07010300, Ministry of Science and Technology of China (2017YFA0206303).

\section{References}

[1] Hou Z P, Yang B C, Wang Y, Ding B, Zhang X M, Yao Y, Liu E K, Xi X K, Wu G H, Zeng Z M, Liu Z Y, Wang W H 2016 Sci. Rep.6 23807

[2] Luo Z, Maassen J, Deng Y X, Du Y C, Garrelts R P, Lundstrom M S, Ye P D and Xu X F 2014 Nat. Commun. 68572

[3] Lee S, Yang F, Suh J, Yang S J, Lee Y, Li G, Choe H S, Suslu A, Chen Y B, Ko C, Park J, Liu K, Li J B, Hippalgaonkar K, Urban J J, Tongay S and Wu J Q 2014 Nat. Commun. 68573

[4] Jain A and McGaughey J H 2015 Sci. Rep. 58501

[5] Qiao J S, Kong X H, Hu Z- X, Yang F, and Jia W 2014 Nat. Commun. 54475

[6] Xia F N, Wang H and Jia Y C 2014 Nat. Commun. 54458

[7] Fei R X, Tran V and Yang L 2015 Phys. Rev. B.91 195319

[8] Baik S S, Kim K S, Yi Y J and Choi H J 2015 Nano lett. 157788

[9] Okajima M, Endo S, Akahama Y and Narita S 1984 Jpn. J. Appl. Phys. 2315

[10] Xiang Z J, Ye G J, Shang C, Lèi B, Wang N Z, Yang K S, Liu D Y, Meng F B, Luo X G, Zou L J, Sun Z, Zhang Y and Chen X H 2015 Phys. Rev. Lett. 115186403

[11] Morita A 1986 Appl. Phys. A. 39227

[12] Kim J M, Baik S S, Ryu S H, Sohn Y, Park S, Park B- G, Denlinger J, Yi Y, Choi H J and Kim K S 2015 Science 349723

[13] Tran V, Soklaski R, Liang Y F and Yang L 2004 Phys. Rev. B. 89235319

[14] Liang L B, Wang J, Lin W Z, Sumpter B G, Meunier V and Pan M H 2014 Nano lett. 146400 [15] Li L K, Yu Y J, Ye G J, Ge Q Q, Ou X D, Wu H, Feng D L, Chen X H and Zhang Y B 2014 Nat. Nanotech. 9372

[16]Liu H, Neal A T, Zhu Z, Luo Z, Xu X F, Tománek D and Ye P D 2014 ACS Nano 84033 
[17] Koenig S P, Doganov R A, Schmidt H, Neto A H C and Özyilmaz B 2014 Appl. Phys. Lett. 104103106

[18] Wang H, Wang X M, Xia F N, Wang L H, Jiang H, Xia Q F, Chin M L, Dubey M and Han SJ 2014 Nano Lett. 146424

[19] Hao C X, Yang B C, Wen F S, Xiang J Y, Li L, Wang W H, Zeng Z M, Xu B, Zhao Z S, Liu Z Y, Tian Y J 2016 Adv. Mater. 283194

[20] Han C Q, Yao M Y, Bai X X, Miao L, Zhu F F, Guan D D, Wang S, Gao C L, Liu C H, Qian D, Liu Y and Jia J- F 2014 Phys. Rev. B. 90085101

[21] Golias E, Krivenkov M and Sánchez-Barriga J 2016 Phys. Rev. B. 93075207

[22] Lüth H Solid Surfaces, Interfaces and Thin films, 4th ed. (Springer, Berlin, 2001)

[23] Zangwill A Physics at Surfaces (Cambridge University Press, Cambridge, 1988)

[24] Kneedler E, Skelton D, Smith K E and Kevan S D 1990 Phys. Rev. Lett. 643151

[25] Aitani M, Hirahara T, Ichinokura S, Hanaduka M, Shin D and Hasegawa S 2014 Phys. Rev. Lett. 113206802

[26] Xiao S H, Wei D H and Jin X F 2012 Phys. Rev. Lett. 109166805

[27] Aitani M, Hirahara T, Ichinokura S, Hanaduka M, Shin D and Hasegawa S 2010 Phys. Rev. B.

82125326

[28] Akahama Y, Endo S and Narita S 1983 J. Phys. Soc. Jpn. 522148

[29] Lima L H D, Barreto L, Landérs R and Siervo A D 2016 Phys. Rev. B. 93035448

[30] Wang Y, Xu G Z, Hou Z P, Yang B C, Zhang X M, Liu E K, Xi X K, Liu Z Y, Zeng Z M, Wang W H and Wu G H 2016 App. Phys. Lett. 108092102

[31] Chang C- Z, Zhang Z C, Feng X, Li K, He K, Wang L- L, Chen X, Dai X, Fang Z, Xue Q- K, Ma X Cand Wang Y Y 2012 Phys. Rev. Lett. 108036805

[32] Liu Y P, Lew W S and Sun L 2011 Phys. Chem. Chem. Phys. 1320208

[33] Tikhonenko F V, Kozikov A A, Savchenko A K and Gorbachev R V 2009 Phys. Rev. Lett. 103 226801

[34] Morozov S V, Novoselov K S, Katsnelson M I, Schedin F, Ponomarenko L A, Jiang D and Geim A K 2006 Phys. Rev. Lett. 97016801

[35] Lang M R, He L, Kou X F, Upadhyaya P, Fan Y B, Chu H, Jiang Y, Bardarson J H, Jiang W J, Chøi E S, Wang Y, Yeh N- C, Moore J and Wang K L 2012 Nano Lett. 1348 
[36] Noriko I, Yusei M, Susumu K, Ichimin S, Minoru K 1985 Chem. Lett. 1119

[37] Du Y C, Neal A T, Zhou H and Ye P D 2016 2D Mater. 3024003

[38] Xia B, Ren P, Sulaev A, Liu P, Shen S- Q and Wang L 2013 Phys. Rev. B. 87085442

[39] Hou Z P, Wang Y, Xu G Z, Zhang X M, Liu E K, Wang W Q, Liu Z Y, Xi X K, Wang W H and Wu G H 2015 Appl. Phys. Lett. 106102102

[40] Hou Z P, Wang Y, Liu E K, Zhang H W, Wang W H and Wu G H 2015 Appl. Phys. Lett. 107 202103

[41] Xu G Z, Wang W H, Zhang X M, Du Y, Liu E K, Wang S G, Wu G H, Liu Z Y and Zhang X X 2014 Sci. Rep. 45709

[42] Chen G L, Han J, Huang T T, Datta S and Janes D B 1993 Phys. Rev. B (R). 474084

[43] Takatsu H, Ishikawa J J, Yonezawa S, Yoshino H, Shishidou T, Oguchi T, Murata K and Maeno Y Phys. Rev. Lett. 2013111056601

[44] Hikami S, Larkin A I and Nagaoko Y 1980 Prog. Theor. Phys. 63707 


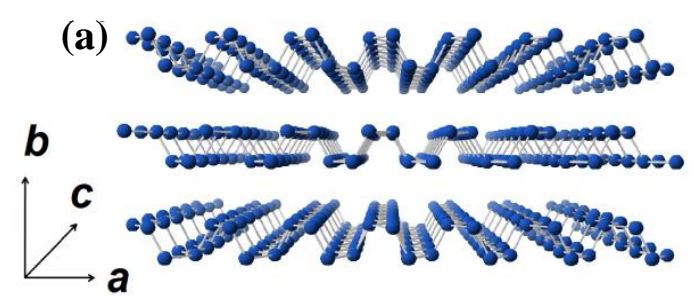

(b)

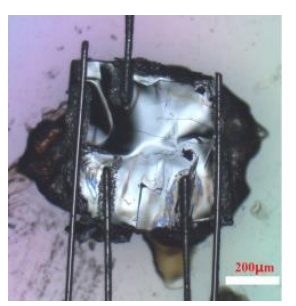

(c)

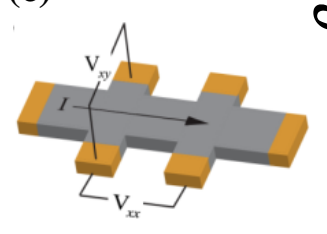

(d)

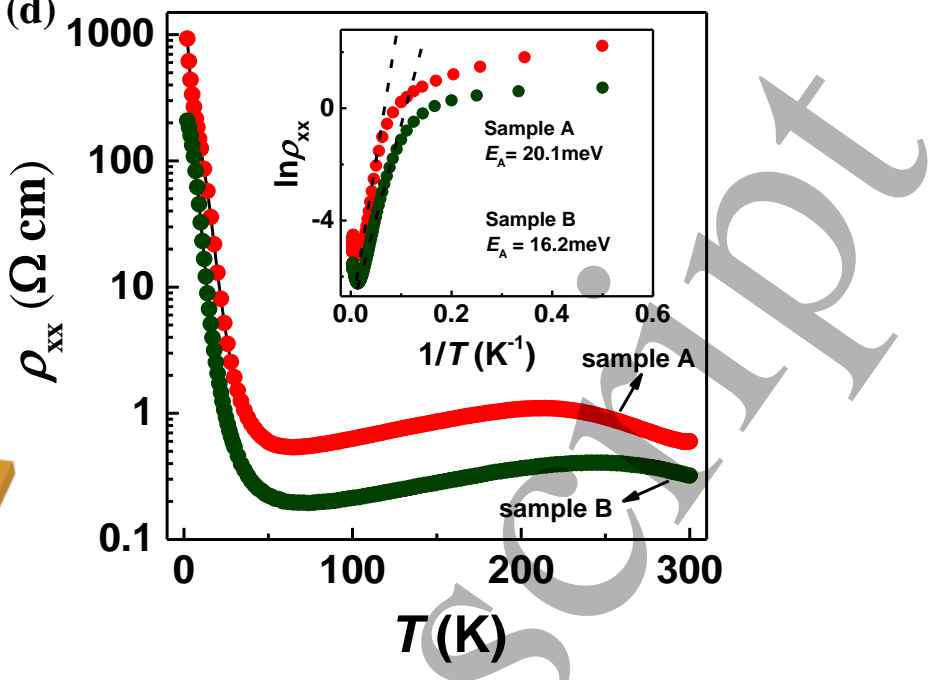

FIG. 1. (a) The perspective side view of the crystal structure of bulk BP. The black arrows show the crystallographic axis of BP (b) Photograph of the devices used for transport measurements. The Hall bar configurations were pasted on the freshly cleaved (0l0) BP plane. (c) The corresponding schematic diagram of the Hall bar configurations. (d) The temperature dependence of $\rho_{\mathrm{xx}}$ in zero-magnetic field for two representative samples, A and B. Inset: the corresponding $\ln \left(\rho_{\mathrm{xx}}\right)$ versus $1 / \mathrm{T}$ plot. The energy gap $E_{\mathrm{g}}$ at low temperatures can be fitted by $\rho_{\mathrm{xx}}(\mathrm{T})=$ constant $\mathrm{x}$ $\exp \left(-E_{\mathrm{g}} / 2 \mathrm{k}_{\mathrm{B}} T\right)$, and the corresponding $E_{\mathrm{g}}$ of the two samples was obtained to be $20.1 \mathrm{meV}$ and $16.2 \mathrm{meV}$ 


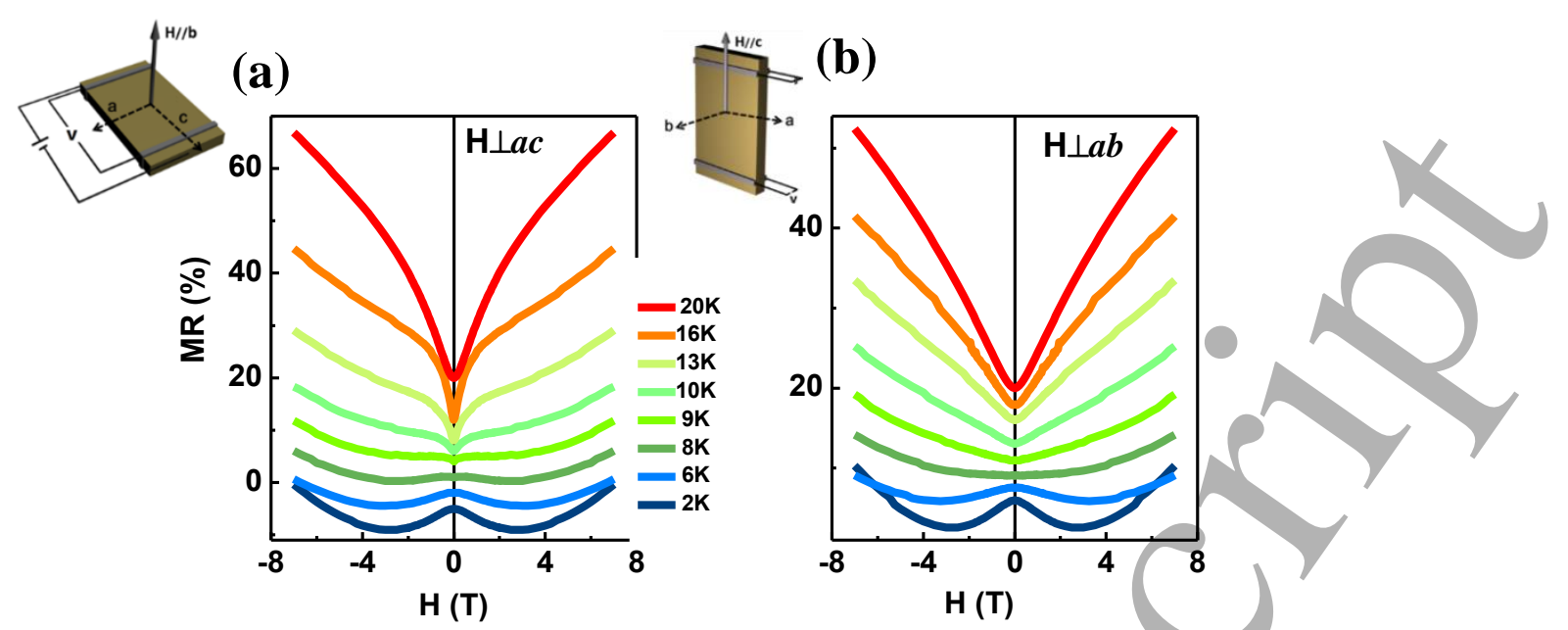

FIG. 2. (a) The magnetic field dependence of MR between $2 \mathrm{~K}$ and $20 \mathrm{~K}$ with current flowing along the $c$-axis (in the $a c$-plane) and magnetic field parallel to the $b$-axis. Each curve is shifted vertically with equidistance to highlight the peculiar WL and WAL crossover driven by the temperature. The WAL and WL cusp was embedded on a broad parabolic or linear background. The inset presents the schematic of Hall bar configurations for measuring $\rho_{\mathrm{xx}}$ (the electrical configuration for measuring Hall resistivity $\rho_{x y}$ is not presented). (b)The field-dependent MR over the temperature range of $2-20 \mathrm{~K}$ with current flowing along the $b$-axis (in the $a b$-plane) and magnetic field parallel to the $c$-axis. The left inset shows the schematic of measurement. 
(a)

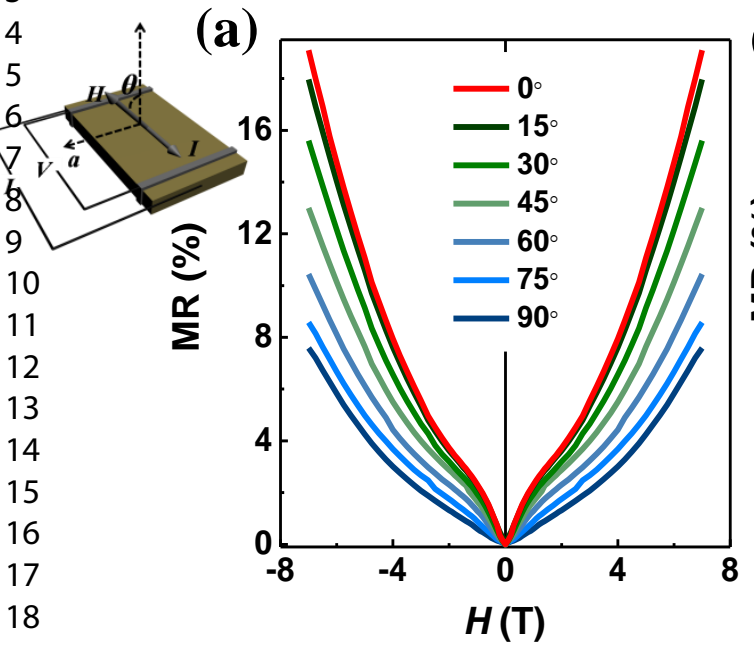

(b)

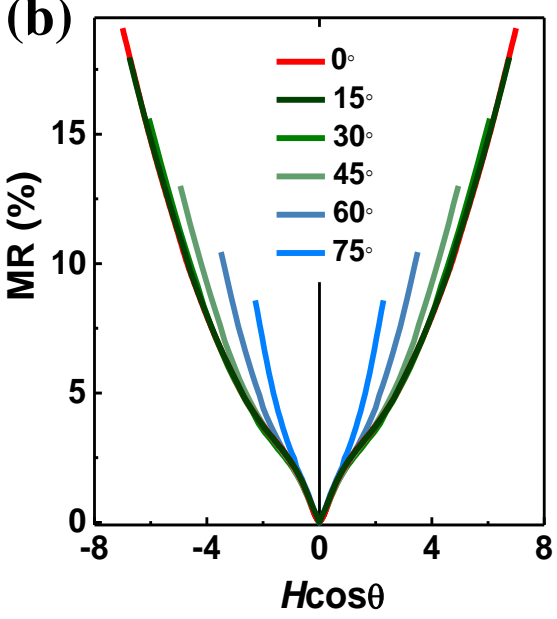

(c)

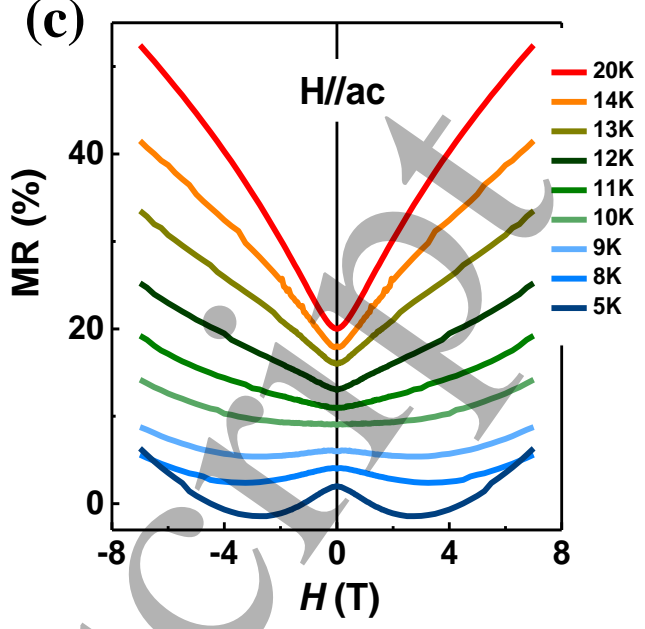

FIG. 3. (a) The magnetic field dependence of MR at 10Kwith magnetic field up to $7 \mathrm{~T}$ at various angles. The left inset shows the configuration of the measurements, where $\theta$ is the angle between the magnetic field and the $b$-axis. (b) The curves of MR as a function of the perpendicular magnetic field component $H \cos \theta$. (c)The field-dependent MR curves at different temperatures at angle $\theta=90^{\circ}$, where $H$ is parallel to the ac-plane. 

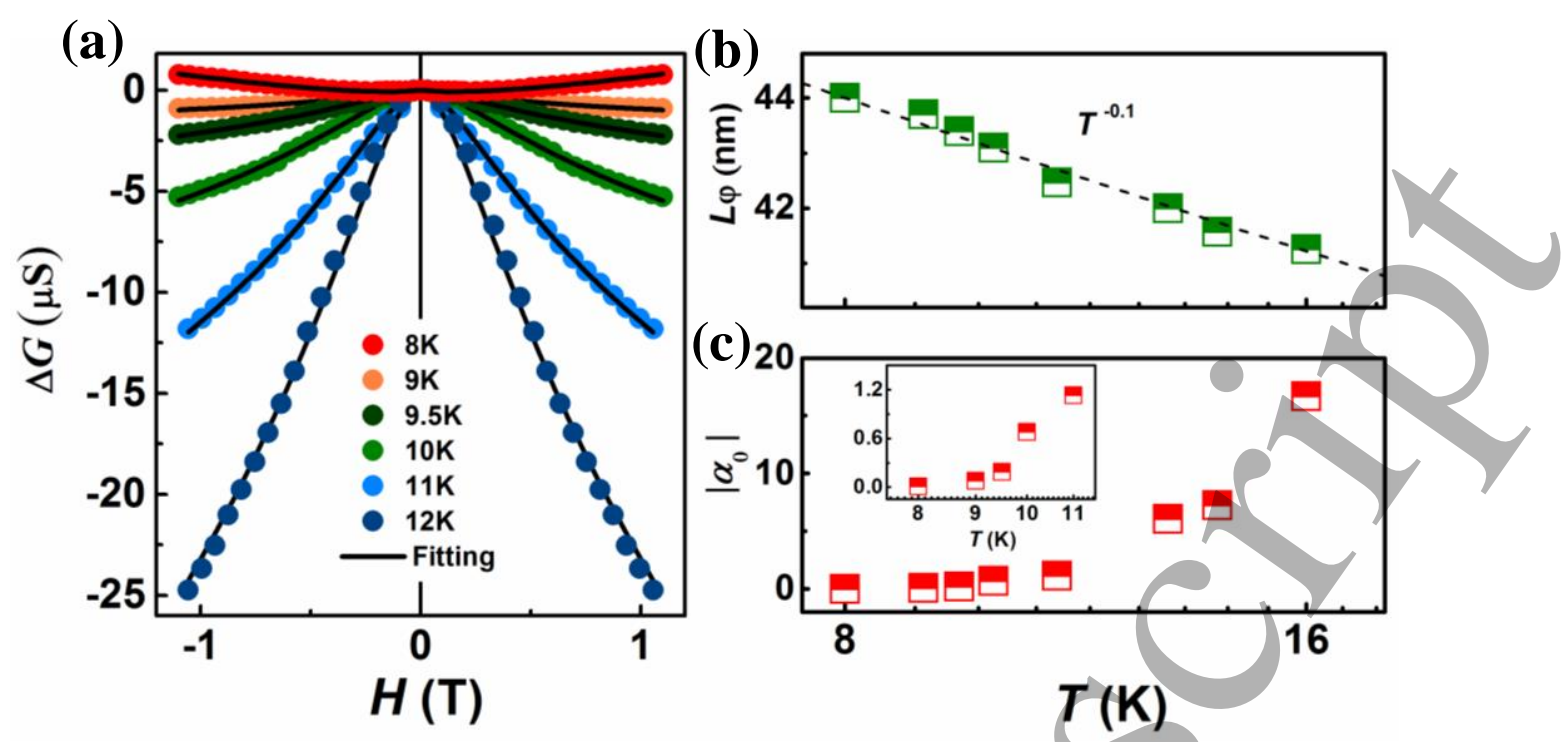

FIG.4. (a) $\Delta G$ curves at a series of temperatures (color circles). The HNL fitting lines are presented as black solid lines. (b) The temperature dependence of $L_{\varphi}$ over the temperature range 8-16 K. (c) The fitted values of $\left|\alpha_{0}\right|$ for WAL over the temperature range 8-16 K. Inset: the values of $|\alpha|$ between $8 \mathrm{~K}$ and $11 \mathrm{~K}$. 